\title{
SPECTRAL MEASURES OF JACOBI OPERATORS WITH RANDOM POTENTIALS
}

\author{
RAFAEL DEL RiO AND LUIS O. Silva
}

\begin{abstract}
Let $H_{\omega}$ be a self-adjoint Jacobi operator with a potential sequence $\{\omega(n)\}_{n}$ of independently distributed random variables with continuous probability distributions and let $\mu_{\phi}^{\omega}$ be the corresponding spectral measure generated by $H_{\omega}$ and the vector $\phi$. We consider sets $\mathscr{A}(\omega)$ which depend on $\omega$, but are independent of two consecutive given entries of the secuence $\omega$, and prove that $\mu_{\phi}^{\omega}(\mathscr{A}(\omega))=0$ for almost every $\omega$. This result is applied to show equivalence relations between spectral measures for random Jacobi matrices and to study the interplay of the eigenvalues of these matrices and their submatrices.
\end{abstract}

Mathematics subject classification (2010): 47B36, 47B39, 47A25, 39A12.

Keywords and phrases: Spectral measures, Jacobi matrices, random potential.

\section{REFERENCES}

[1] N.I. AkHiEZer, AND I.M. Glazman, Theory of linear operators in Hilbert space, Dover Publications Inc., New York, 1993.

[2] JU.M. BEREZANSKII, Expansions in eigenfunctions of selfadjoint operators, Translations of Mathematical Monographs 17, American Mathematical Society, Providence, RI, 1968.

[3] R. CARmona, And J. Lacroix, Spectral theory of random Schrödinger operators, Probability and its Applications, Birkhäuser, Boston, 1990.

[4] H. CyCon, R. Froese, W. Kirsch, AND B. Simon, Schrödinger operators with application to quantum mechanics and global geometry, Texts and Monographs in Physics, Springer-Verlag, Berlin, 1987.

[5] S. Denisov, AND B. Simon, Zeros of orthogonal polynomials on the real line, J. Approx. Theory, 121, 2 (2003), 357-364.

[6] L. Fu, AND H. Hochstadt, Inverse theorems for Jacobi matrices, J. Math. Anal. Appl., 47 (1974), $162-168$.

[7] F. GeSZTESY, AND B. Simon, $m$-functions and inverse spectral analysis for finite and semi-infinite Jacobi matrices, J. Anal. Math., 73 (1997), 267-297.

[8] A.Y. Gordon, AND A.S. Kechris, Measurable enumeration of eigenelements, Appl. Anal., 71, 1-4 (1999), 41-61.

[9] V. JAKŠIĆ, AND Y. LAST, Spectral structure of Anderson type Hamiltonians, Invent. math, 141, 3 (2000), 561-567.

[10] T. KATO, Perturbation theory for linear operators. Classics in Mathematics. Springer-Verlag, Berlin, 1995.

[11] S. Naboko, I. PChelintseVa, And L.O. Silva, Discrete spectrum in a critical coupling case of Jacobi matrices with spectral phase transitions by uniform asymptotic analysis, J. Approx. Theory, 161, 1 (2009), 314-336.

[12] L. Pastur, And A. Figotin, Spectra of random and almost-periodic operators, Grundlehren der Mathematischen Wissenschaften 297, Springer-Verlag, Berlin, 1992.

[13] W. Rudin, Real and complex analysis, Third edition, McGraw-Hill, New York, 1987.

[14] B. Simon, Orthogonal polynomials on the unit circle, Part 1, American Mathematical Society Colloquium Publications 54,1, American Mathematical Society, Providence, RI, 2005. 
[15] G. TESCHL, Jacobi operators and completely integrable nonlinear lattices, Mathematical Surveys and Monographs 72, American Mathematical Society, Providence, RI, 2000. 